\title{
Nonorganic Hearing Loss: A Case Profile
}

\author{
Archana Rai Sarve ${ }^{1}$ Hima Pakkath Venu ${ }^{1} \quad$ Mariya Shaju ${ }^{1}$ \\ ${ }^{1}$ Nitte Institute of Speech and Hearing, Mangalore, Karnataka, India \\ Address for correspondence Archana Rai Sarve, Nitte Institute of \\ Speech and Hearing Medical Sciences Complex, Deralakatte, Mangalore, \\ Karnataka 575018, India (e-mail: archana.rai04@gmail.com).
}

\begin{abstract}
Keywords

- functional hearing loss

- children

- pure tone audiogram

- auditory brainstem

response

- otoacoustic emission

Adults and children exhibit nonorganic hearing loss (NOHL). In children, NOHL can be due to several underlying factors. Issues of this kind if not treated on time may become risky for the child's well-being if the underlying cause of NOHL is psychosocial and emotional. In most of the clinical settings attention is less drawn toward diagnosis and management of the root cause of NOHL. In this present article, we present the audiological profile of a 7-year-old girl with NOHL. The patient came to the department with the compliant of difficulty in hearing. Immittance results showed no abnormalities; however, reliable pure tone results could not be obtained. Subjective tests revealed bilateral hearing loss and objective assessment indicated a normal hearing. On the second visit, audiometric tests were repeated which showed normal hearing. After exclusion of neurological pathology and psychological consultation, the diagnosis of NOHL was stated and the girl was scheduled for regular appointments with a psychologist. In addition, literature on the causative factors related to NOHL has also been discussed in the present article.
\end{abstract}

\section{Introduction}

Functional hearing loss (FHL) can be described as hearing loss appearing in hearing tests with no corresponding physical impairment in the auditory system. FHL or nonorganic hearing loss (NOHL) can be seen in children as well as adults, which can be the result of conscious malingering or may be psychogenic in nature. Within the pediatric population, prevalence of NOHL is reported to be approximately 5 to $7 \% .{ }^{1}$ Children typically exhibit pediatric nonorganic hearing loss (PNOHL) between the age of 6 and 19 years with peak occurrence observed at the age of 11 years. It is also reported that girls are affected twice as often as boys. ${ }^{1,2}$ The major causes of NOHL in children includes poor academic performance, citing hearing loss as an excuse for not doing school work or to fulfill their basic underlying needs, significant emotional problems such as hostility toward the parents, and inability to deal with peer group. As stated, ${ }^{3}$ children presenting with PNOHL may have a basic underlying need that is not fulfilled and may choose from a variety of symptoms ranging from conscious to psychosomatic, to express that need. PNOHL can also be symptomatic of underlying psychosocial stressors. These issues, left unresolved, may become harmful to a child's well-being, especially if the hearing loss is replaced with functional symptoms that are emotionally and psychosocially damaging to the child. In most clinical settings, attention is rarely focused on determining the underlying problems leading to PNOHL or to the management of the condition.

NOHL is characterized by a discrepancy between pure tone audiometry thresholds and normal speech discrimination. Children with PONHL usually show no evidence of difficulty in hearing during normal conversational speech or outside the testing condition., ${ }^{2,4}$ The recommended audiological management of NOHL in children comprises history taking, diagnosis, and audiological and psychological counseling. According to the literature, prognosis depends on the severity of the patient's school and/or personal problems.

\section{Case Report}

A 7-year-old girl came to the department of Audiology and Speech Language pathology with the complaint of reduced hearing and difficulty in understanding what the teachers say in classes. The child's mother reported that hearing difficulty was noticed since last 6 months from the date of 
consultation. Upon detailed examination the mother reported that the child was attending regular school and was studying in $6^{\text {th }}$ standard class. She also reported that the child performed excellently in academics and was ambitious too. However, after her father left for another country to support the family financially the child started to show issues related to hearing. On informal assessment speech and language development was found to be age appropriate, and no significant medical history was noted.

Otological examination by the medical professionals revealed no pathological condition of middle ear. Detailed audiological evaluation was performed which included basic audiometric tests, i.e., tympanometry, acoustic reflex thresholds, pure-tone audiometry (PTA) and speech audiometry. The impedance results revealed bilateral 'A' type tympanogram with ipsilateral acoustic reflexes present for $500 \mathrm{~Hz}, 1$ $\mathrm{kHz}, 2 \mathrm{KHz}$ and $4 \mathrm{kHz}$, indicative of absence of any middle ear pathological conditions. PTA revealed moderate to moderately severe hearing loss ( - Fig. 1); however, the thresholds were highly inconsistent and not reliable. In addition, due to the same issues air conduction (AC) and bone conduction (BC) masking could not be performed. Speech audiometric results revealed speech recognition threshold as $<20 \mathrm{~dB}$ bilaterally and discrimination scores were found to be $100 \%$ bilaterally, which was contradicting with the PTA results. Due to the poor correlation between the results of the different tests, additional tests including otoacoustic emissions (OAE) and auditory brainstem response (ABR) were administered. Distortion product OAE (DPOAE) was performed and bilateral DPOAE's were present suggestive of normal outer hair cell functioning. Threshold estimation was done using $A B R$ for $500 \mathrm{~Hz}$ tone burst and click stimulus. ABR peak $V$ was present up till $30 \mathrm{dBnHL}$ which clearly indicated hearing sensitivity within normal limits.

As there was a mismatch in the results of the above tests and there was no possible organic cause that was ruled out, the next step was to administer the confirmatory tests to identify the NOHL. The tests required the patient's cooperation for accurate results, so the child was gently instructed that her cooperation is a basis of obtaining adequate results and she should respond as precisely and accurately as she could. The tests administered included YES or NO test, Pulse Count Test, and Lambord test.

YES or NO test result revealed hearing sensitivity within normal limits, as the child was responding to the tone which was below the admitted threshold of PTA ( - Fig. 2). In pulse count test 10 stimuli were presented above the admitted threshold of the child and 10 pulses were presented below the admitted threshold. However child could count all the 20 pluses which were presented which again is inductive that the admitted thresholds are not appropriate. Lambord test results indicated that as the noise intensity increases the vocal intensity also changed, vocal intensity changes were observed even before the noise reached the admitted thresholds. Once a set of confirmatory test were done it was found that there was no organic cause for hearing loss.

Interactive session was performed with the patient and parents individually. On enquiry, the child stated she was burdened with daily chore works and the delivery of instruction by the mother to her was unpleasant. Hence, to avoid this patient acted to have hearing loss. On the basis of all the above motioned examinations no organic cause of hearing loss was found. Parents were counseled about the problem and were recommended to psychological assessment and management. It was explained that separation from the father has created a significant influence on the emotional state of the child and resulting in the reported hearing problem. Also the child was scheduled for regular psychological counseling.

\section{Discussion}

Uncommon informal behavior as well as discrepancies between different tests should suggest NOHL which must be completely evaluated. Review of literature on NOHL states that there are only few cases documented, ${ }^{5-7}$ which
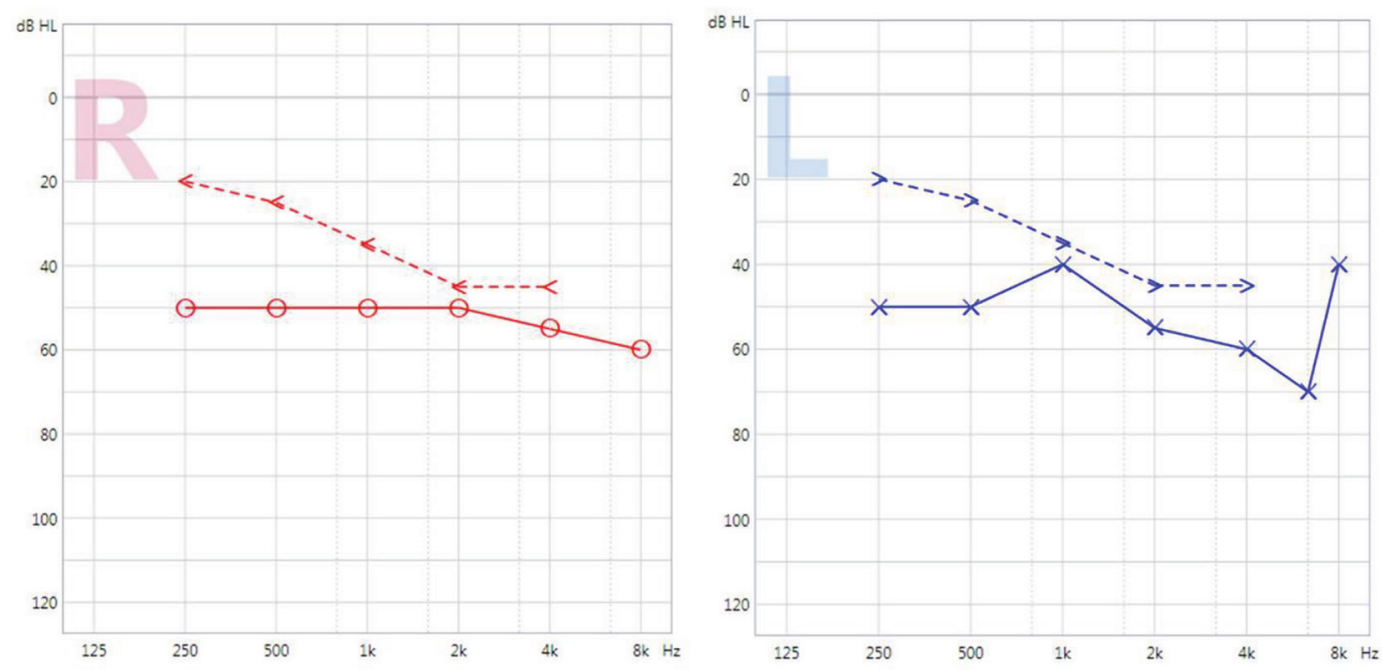

Fig. 1 Pure tone thresholds of the patient during the first testing. Red indicates right ear \& blue indicates left ear. 

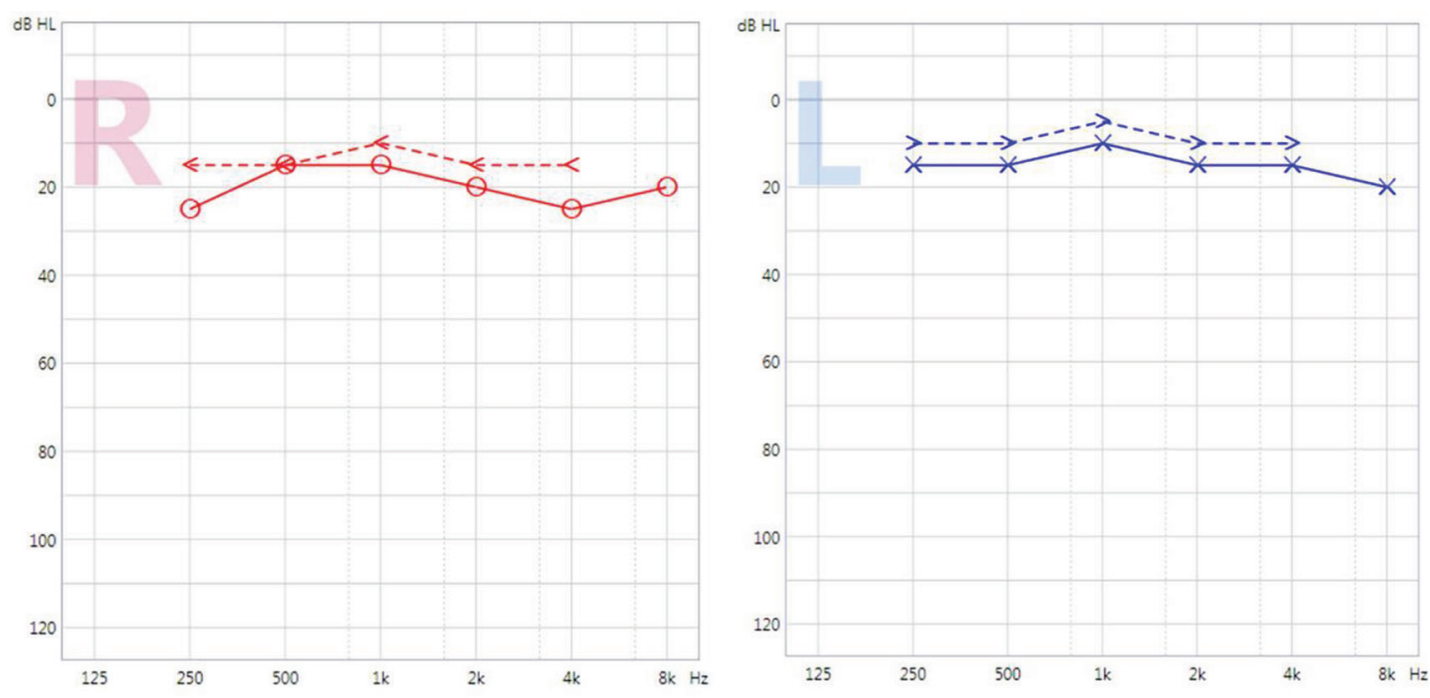

Fig. 2 Pure tone thresholds of the patient during the second testing. Red indicates right ear \& blue indicates left ear.

are diagnosed with NOHL. According to a study over a period of six years (2003-2008) the diagnosed cases of NOHL in children is only $0.1 \%$ out of 19,353 cases. ${ }^{5}$ Combining objective and subjective hearing tests constitutes a clue component of effective management.

When NOHL is suspected, a test battery approach should be followed and should be extended to speech audiometry, otoacoustic emission, and ABR. Due to the advances in objective audiometry, the diagnosis of NOHL is less challenging than management and counseling. ${ }^{8}$ Diagnostic steps proposed include correlating speech audiometry, ABR, and TEOAE with (transient evoked otoacoustic emission) retest PTA.

Deficit of adequate diagnosis may lead to unnecessary expenses and negative consequences, such as steroid treatment and hearing aid fitting. The genuine cause of the complaint remains unknown in case of misdiagnosis. Identifying the problem and counseling the parents regarding the same is an essential part of management on NOHL in children. Meanwhile differential diagnosis from ANSD (auditory neuropathy spectrum disorder) and CAPD (central auditory processing disorder) is important.

\section{Conclusion}

Assessment of individuals with FHL is not something that an audiologist encounters frequently in their day to day practices. On the other hand, there is no standard protocol available concerning the diagnosis of NOHL in pediatric population. FHL could stem from many causes. Moreover due to various factors, children could have personal or academic issues leading to symptoms like FHL. Hence drawing conclusion only from audiological findings is not a wise choice. A multidisciplinary approach must be followed involving evaluation from different disciplines; one of the most important evaluations is from the child psychologist which reveals the root cause of the child's behaviors.

\section{Conflict of Interest}

None declared.

\section{Acknowledgment}

The authors extend their gratitude to the Director, Nitte Institute of Speech and Hearing for permitting us to carry out this research. We also thank the Principal, Nitte Institute of Speech and Hearing for her constant support. We sincerely express our profound thanks to the entire faculty at the Department of Othorhinolaryngology and Psychology, Justice K S Hedge Hospital, Mangaluru for their unconditional help.

\section{References}

1 Rintelmann W, Harford E. The detection and assessment of pseudohypoacusis among school-age children. J Speech Hear Disord 1963;28:141-152

2 Dixon RF, Newby HA. Children with nonorganic hearing problems. AMA Arch Otolaryngol 1959;70(5):619-623

3 Northern JL, Downs MP. Hearing in Children. Lippincott Williams \& Wilkins; 2002

4 Gelfand SA. Physiological methods in audiology. Ess Audiolog 2001; 367-369

5 Holenweg A, Kompis M. Non-organic hearing loss: new and confirmed findings. Eur Arch Otorhinolaryngol 2010;267(8):1213-1219

6 Saravanappa N, Mepham GA, Bowdler DA. Diagnostic tools in pseudohypacusis in children. Int J Pediatr Otorhinolaryngol 2005;69(9):1235-1238

7 Morita S, Suzuki M, Iizuka K. Non-organic hearing loss in childhood. Int J Pediatr Otorhinolaryngol 2010;74(5):441-446

8 Drouillard M, Petroff N, Majer J, Perrot C, Quesnel S, François M; ORL et CCF, CHU Robert Debré, Paris, France. Pseudohypacusis in children: circumstances and diagnostic strategy. Int J Pediatr Otorhinolaryngol 2014;78(10):1632-1636 